\title{
Electron-Beam Manipulation Techniques in the SINBAD Linac for External Injection in Plasma Wake-Field Acceleration
}

B. Marchetti, R. Aßmann, C. Behrens, R. Brinkmann, U. Dorda, K. Flöttmann, I. Hartl, M. Hüning, Y. Nie, H. Schlarb, J. Zhu

EAAC 2015, Isola D'Elba, 15/09/2015

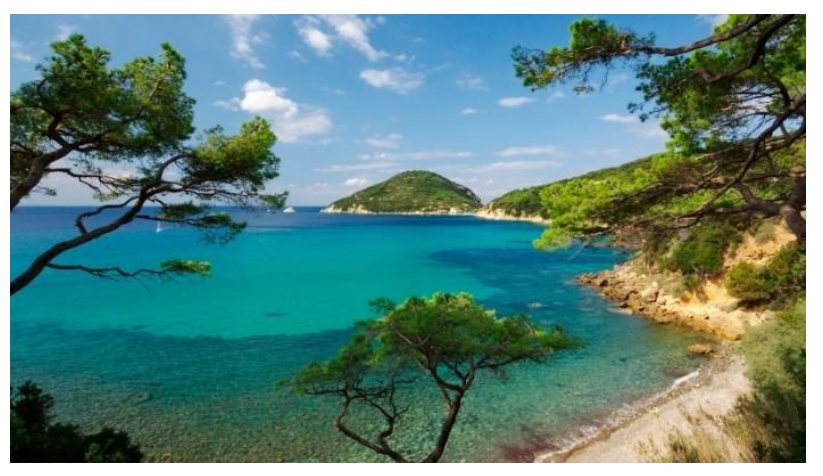




\section{Outlook}

$>$ Overview SINBAD/SINBAD linac:

- SINBAD linac as an experiment itself

- Linac requirements for future experiments

$>$ Linac layout

- Stage 1 - baseline

- Stage 2 - upgrade

$>$ Overview of the e-bunch compression techniques

- Pure velocity bunching

- Velocity bunching + bunch re-compression in the dogleg

- Magnetic compression with slit-cut

- Tolerances

$>$ Conclusions and summary 


\section{SINBAD}

\section{$S I N B A D=$ and Aacievertes of $\mathrm{D}-\mathrm{y}$}

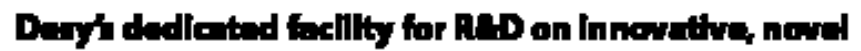

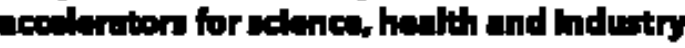

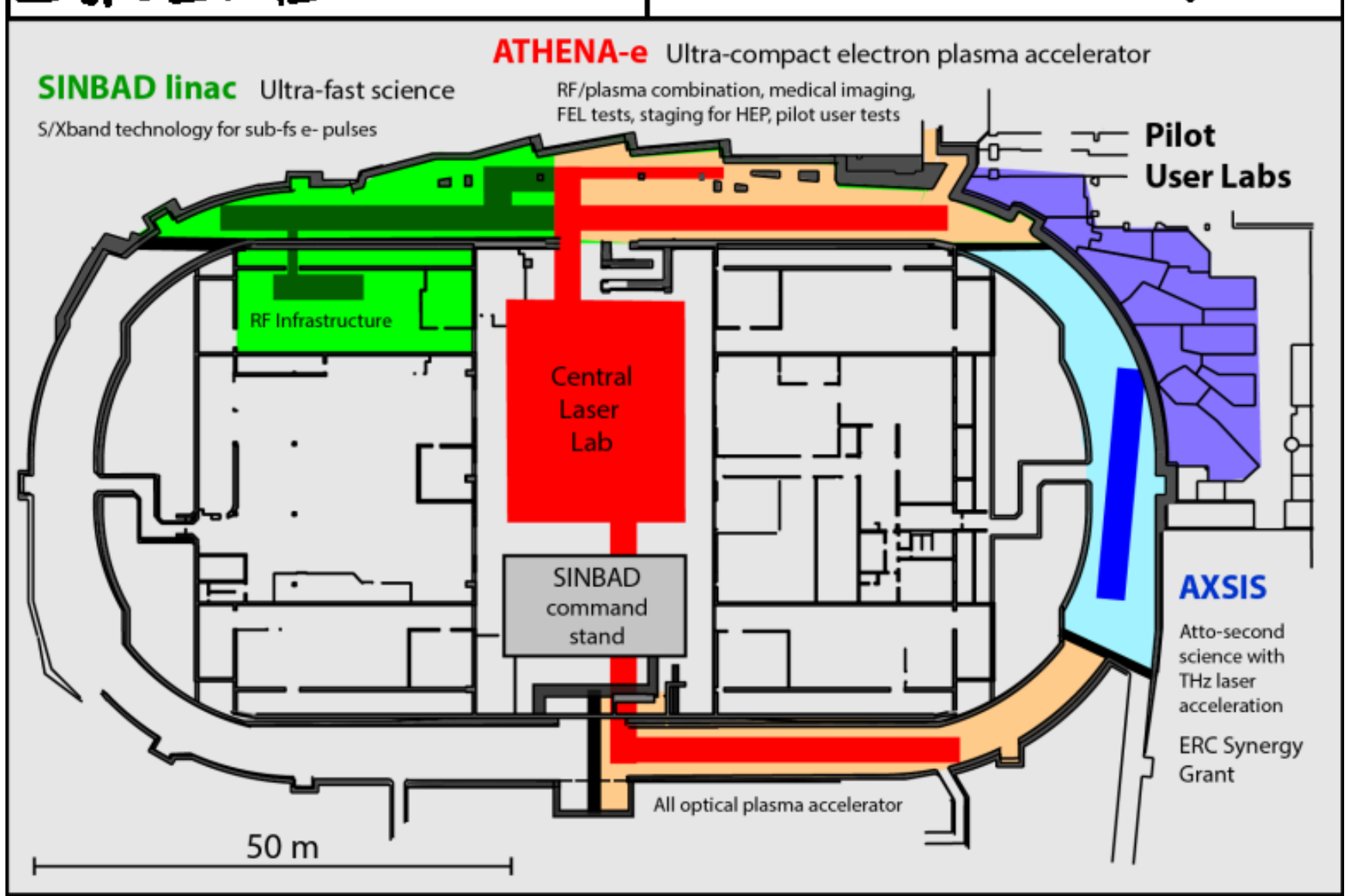

\section{SINBAD}

Short INnovative Bunches and Accelerators at Desy

PL: Ulrich Dorda

Dedicated multipurpose accelerator R\&D facility with several experiments from ulltra-fast science and high gradient accelerator modules.

Talk by U. Dorda in Session 1 WG4. 


\section{SINBAD linac as an experiment itself}

- Goal: production \& characterization of ultra-short bunches (tFWHM $\leq 1 \mathrm{fs})$

$\rightarrow$ Charge as high as possible

$\rightarrow$ Relaxed transverse spot-size $(>100 \mu \mathrm{m})$

$\rightarrow$ Mainly technical challenges

Challenges in beam
diagnostics:
$\begin{aligned} & \text { Low charge: } \\ & \text { - Low signal/noise ratio } \\ & \text { some diagnostics not yet } \\ & \text { development }\end{aligned}$
$>$ Sub-fs longitudinal resolution is
needed : state of the art are
Xband TDS with $1 \mathrm{fs}$
resolution.

\section{Challenges in synchronization: \\ $>$ Short signal, fs level synchronization is needed (both RF-RF and laser- $\mathrm{RF}$ ).}




\section{SINBAD linac as an experiment itself}

- Goal: production \& characterization of ultra-short bunches (tFWHM $\leq 1 \mathrm{fs})$

$\rightarrow$ Charge as high as possible

$\rightarrow$ Relaxed transverse spot-size $(>100 \mu \mathrm{m})$

$\rightarrow$ Mainly technical challenges

Much experience at DESY thanks to European XFEL, REGAE etc...

Just few examples:

- G. Kube et al., "Transverse Beam Profile Imaging of Few-Micrometer Beam Sizes Based on a Scintillator Screen", Proc. IBIC 2015.

- S. Bayesteh, „Transverse electron beam diagnostics at REGAE“, PhD thesis (2014).

- M. Hansli et al. „A BEAM ARRIVAL TIME CAVITY FOR REGAE AT DESY“, Proc. of IPAC 2014

- Low signal/noise ratio

- some diagnostics not yet available/under development

Sub-fs longitudinal resolution is needed : state of the art are Xband TDS with $1 \mathrm{fs}$ resolution.
Challenges in synchronization:

$>$ Short signal, fs level synchronization is needed (both RF-RF and laser$\mathrm{RF})$.
- M. Hoffmann et al., "HIGH SPEED DIGITIAL LLRF FEEDBACKS FOR NORMAL CONDUCTING CAVITY OPERATION”, Proc. Of IPAC 2014

- M. Titberidze et al., „PRESENT AND FUTURE OPTICAL-TO-MICROWAVE SYNCHRONIZATION SYSTEMS AT REGAE FACILITY FOR ELECTRON DIFFRACTION AND PLASMA ACCELERATION EXPERIMENTS", Proc. IPAC 2015.

- J. Mueller at al., „ALL-OPTICAL SYNCHRONIZATION OF PULSED LASER SYSTEMS AT FLASH AND XFEL“, Proc. IPAC 2015

... And many more. 


\section{SINBAD linac - general philosophy for future experiments}

- Who will be the „users" of the SINBAD linac?

$\rightarrow$ Experiments involving Novel High Gradient Acceleration Techniques: e.g. LPWA, Dielectric Wake-Field Acceleration, THz laser acceleration in dielectric-loaded structures..

- What types of e-beam will such experiments need?
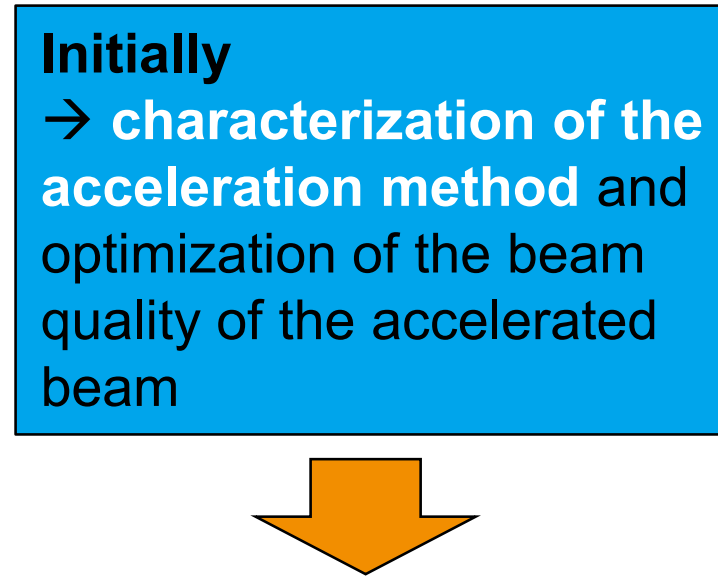

Ultra-short probes $\rightarrow$ time resolution Ultra-high stability $\rightarrow$ synchronization Small transverse focus (tens of $\mu \mathrm{m}$ - few $\mu \mathrm{m})$
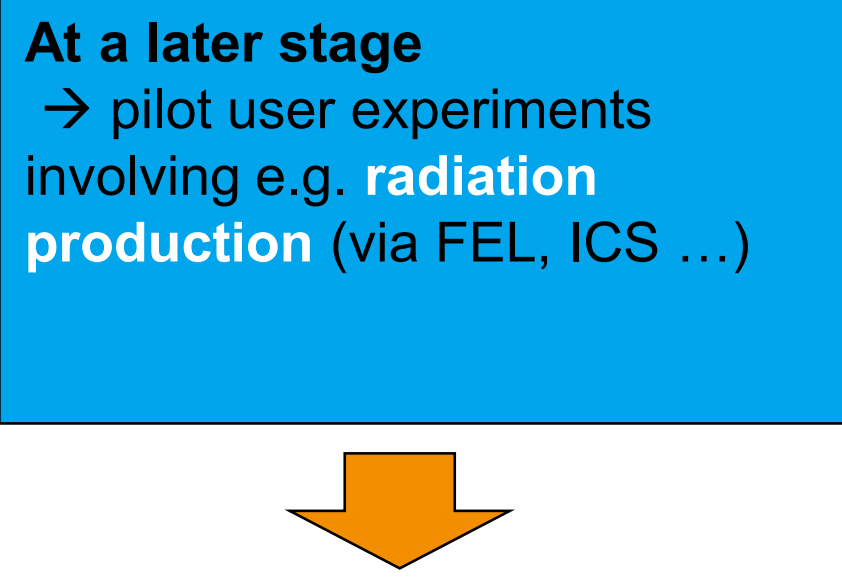

- Sufficiently high brightness $\rightarrow$ radiation generation

- The e-bunch duration has to be tuned, according to the requirements for the production of radiation. 


\section{External injection into LWFA with usable beam quality Requirements for the injected e-bunches}

$>$ Energy $~ 100 \mathrm{MeV}$ (less de-phasing issues for $\mathrm{n} \sim 10^{16}-10^{17} \mathrm{~cm}^{-3}$ )

$>$ Bunch length $<5$ fs (small final energy spread)

$>$ Time jitter stability $<10$ fs

$>$ Small transverse spot-size: matched $B$ functions range from $\mathrm{cm}\left(\mathrm{n} \sim 10^{14}\right)$ to $\mathrm{mm}\left(\mathrm{n} \sim 10^{17}\right)$

Example: Simulations at $\mathrm{n}=10^{17}$
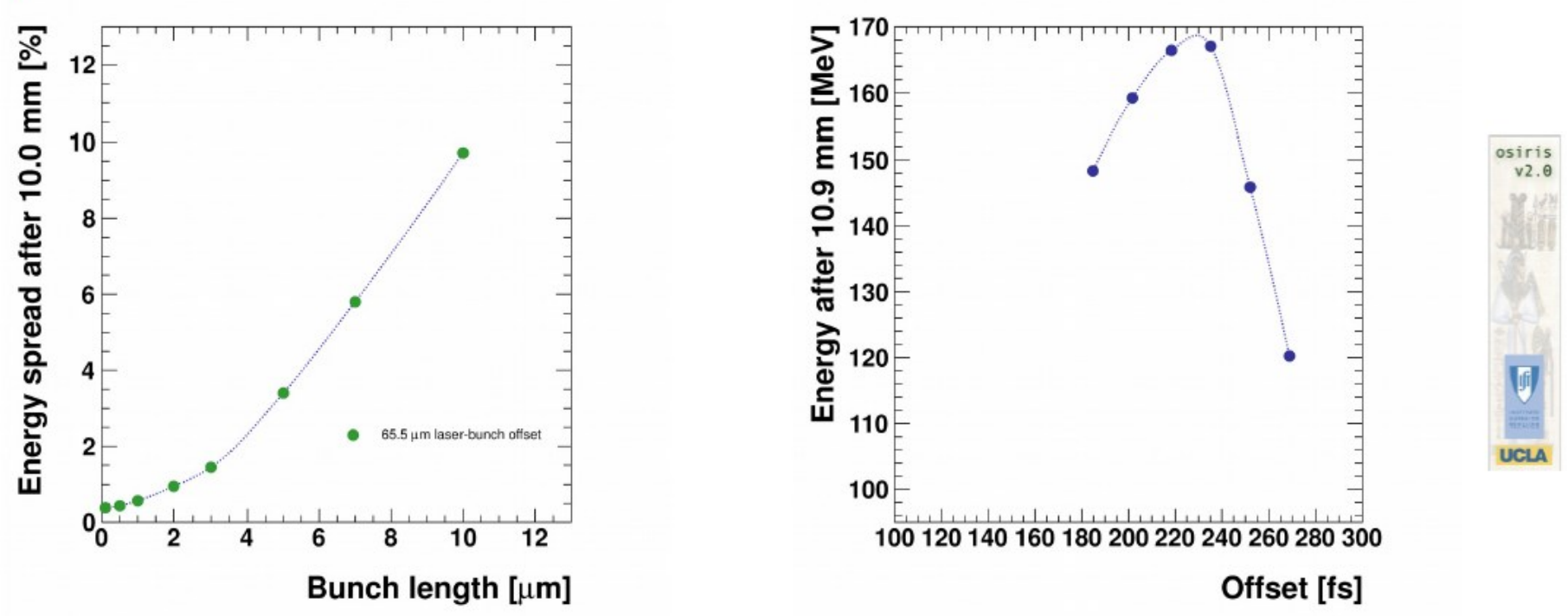

- R. Assmann, J. Grebenyuk, TUOBB01, Proceed. IPAC 2014.

Simulations by J. Grebenyuk

- J. Grebenyuk et al., TUPME064, Proceed. IPAC 2014 


\section{SINBAD LINAC layout - baseline}

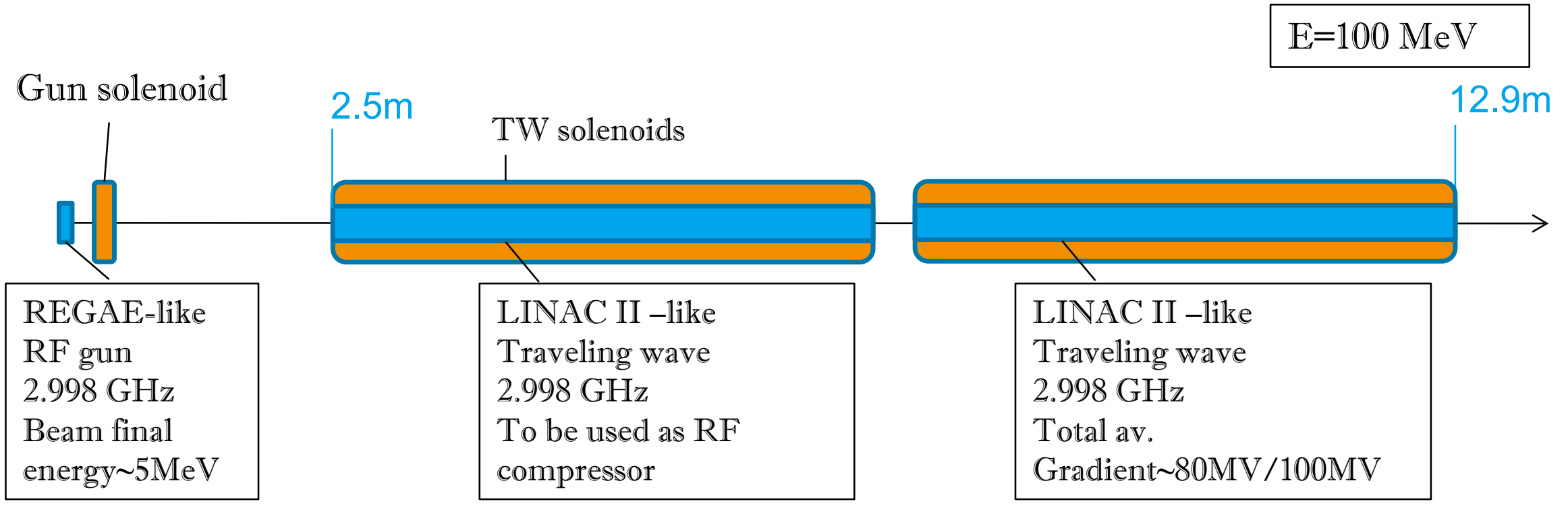

Special attention is being given to the flexibility and the stability of the elements:

- Load-lock system for cathode exchange (Cs2Te for laser pulse length $\geq 1 \mathrm{ps}$, metallic cathodes for shorter laser pulses)

- 2 gun solenoids respectively for low charge/high charge WPs (in the sketch above only the one for the low charge WP is shown)

- Flexible photo-cathode laser system: Yb doped mJ laser with tunable pulse duration 190fs10ps.

- Each RF cavity fed by one klystron. No SLEDs. 


\section{SINBAD LINAC layout - upgrade}

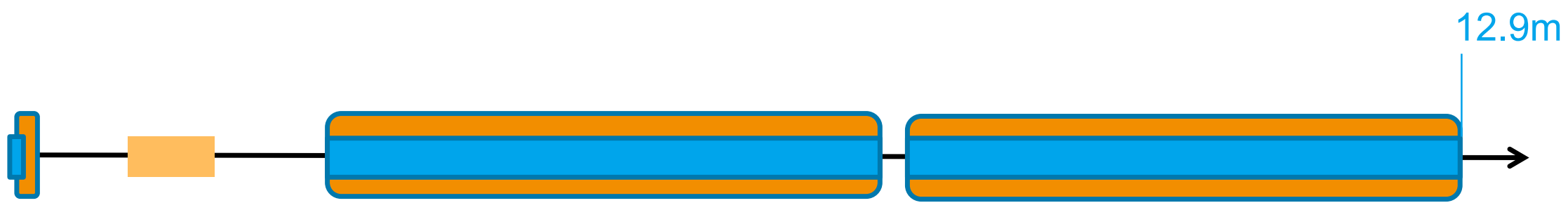

Xband linearizer

TW

$11.9942 \mathrm{GHz}$

Total av.

Gradient $6 \mathrm{MV}$

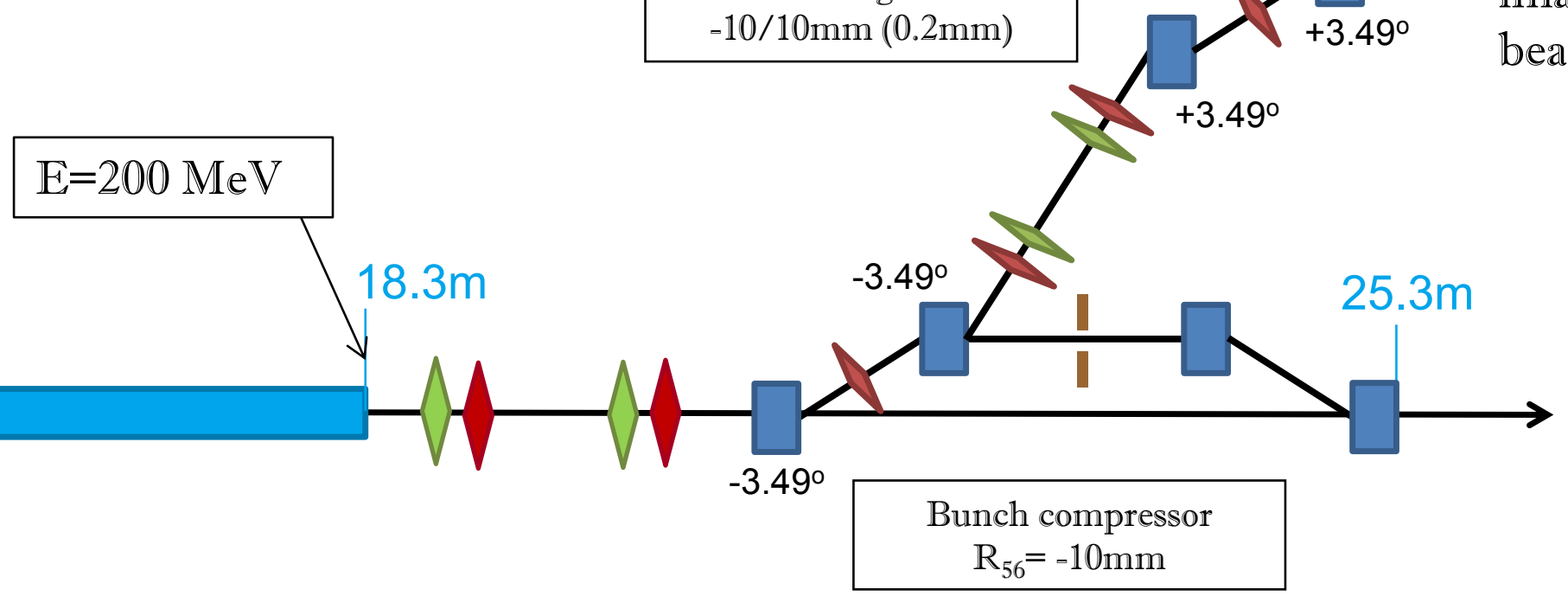

To the plasma exp. and diagnostics beam-line 


\section{VELOCITY BUNCHING}

- L. Serafini and M. Ferrario, AIP Conf. Proc. 581, 87-106 (2001).

- S.G. Anderson et al. PRSTAB 8014401 (2005).

- M. Ferrario et al. PRL 104054801 (2010).

- A. Bacci, A.R.Rossi NIM A 740 (2014) 42-47.

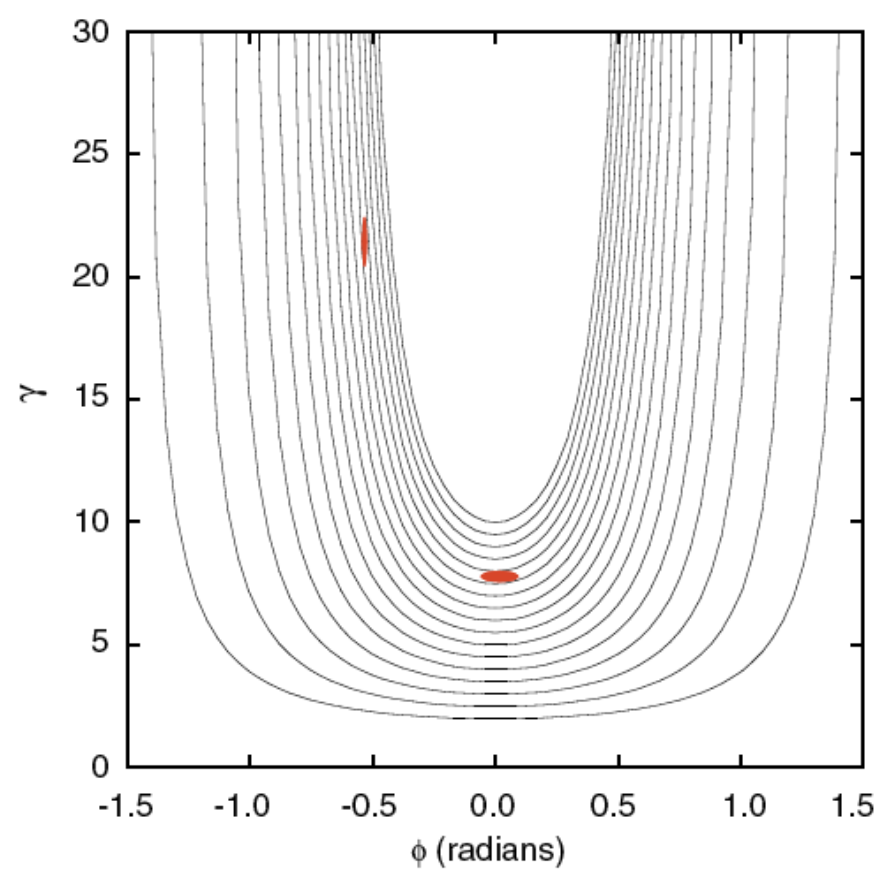

Pro: very good transverse emittance, no CSR, no charge loss, small spot size at the exit

Contra: tight phase tolerances on the RF compressor, long. non-linearity
Electron Bunch from RF injector Initial velocity $\beta_{0} \sim 0.994(4 \mathrm{MeV})$

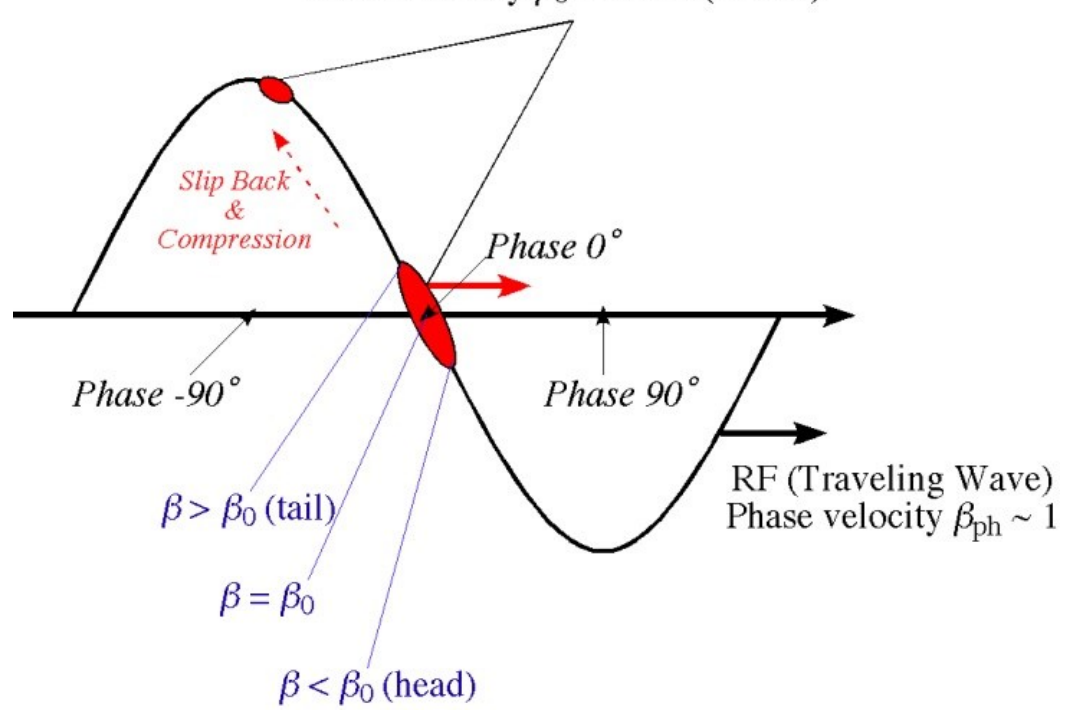




\section{VELOCITY BUNCHING}
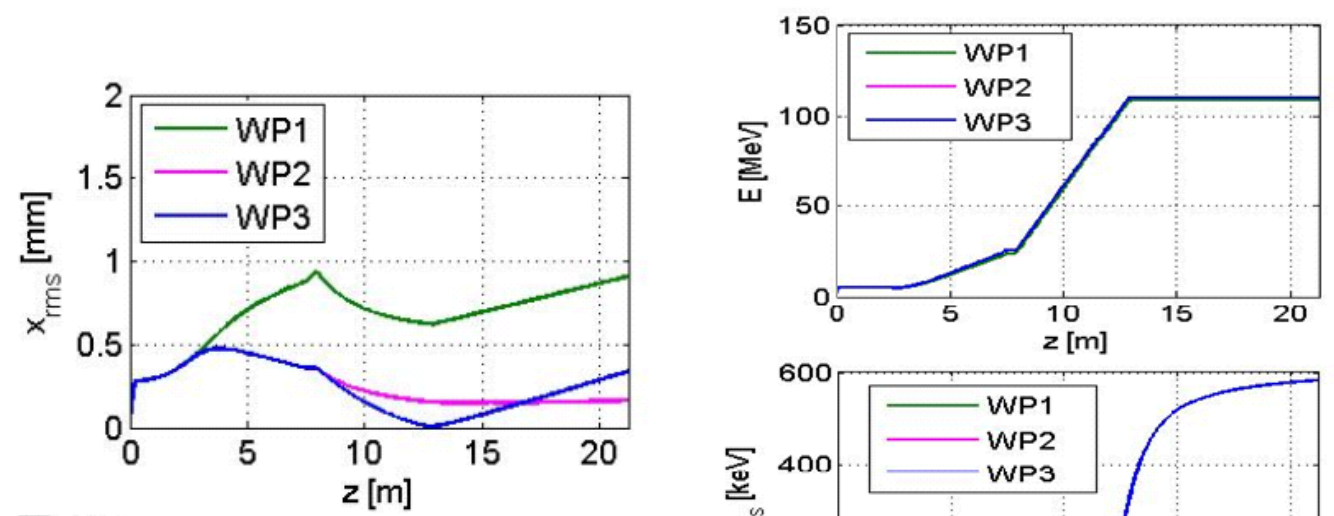

\section{Examples of WPs providing different final spot-sizes.}

Table 1: Low Charge Working Points at Z1
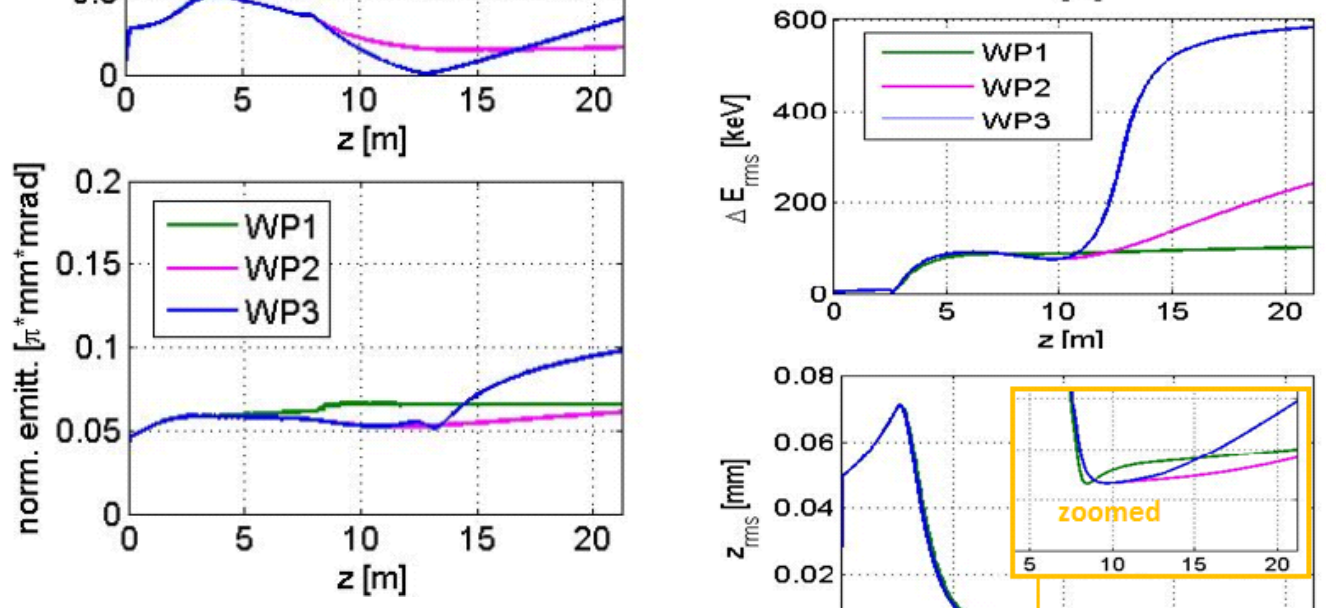

\begin{tabular}{lccc}
\hline Parameter & WP1 & WP2 & WP3 \\
\hline Charge $[\mathrm{pC}]$ & 0.5 & 0.5 & 0.5 \\
FWHM [fs] & 2.1 & 2.7 & 4 \\
E $[\mathrm{MeV}]$ & 109 & 111 & 111 \\
$\Delta E / E[\%]$ & 0.1 & 0.1 & 0.3 \\
$\sigma_{x, y}[\mathrm{~mm}]$ & 0.6 & 0.15 & 0.009 \\
n $\epsilon_{x, y}[\mu \mathrm{m}]$ & 0.07 & 0.05 & 0.05 \\
$I_{p}$ (in 1 FWHM) $[\mathrm{A}]$ & 133 & 115 & 87 \\
\hline
\end{tabular}

B. Marchetti et al. , TUPWA030,

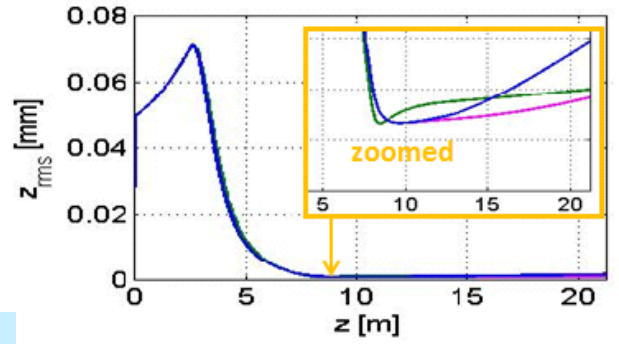

Proc. IPAC 2015.

To the medical imaging beam-line

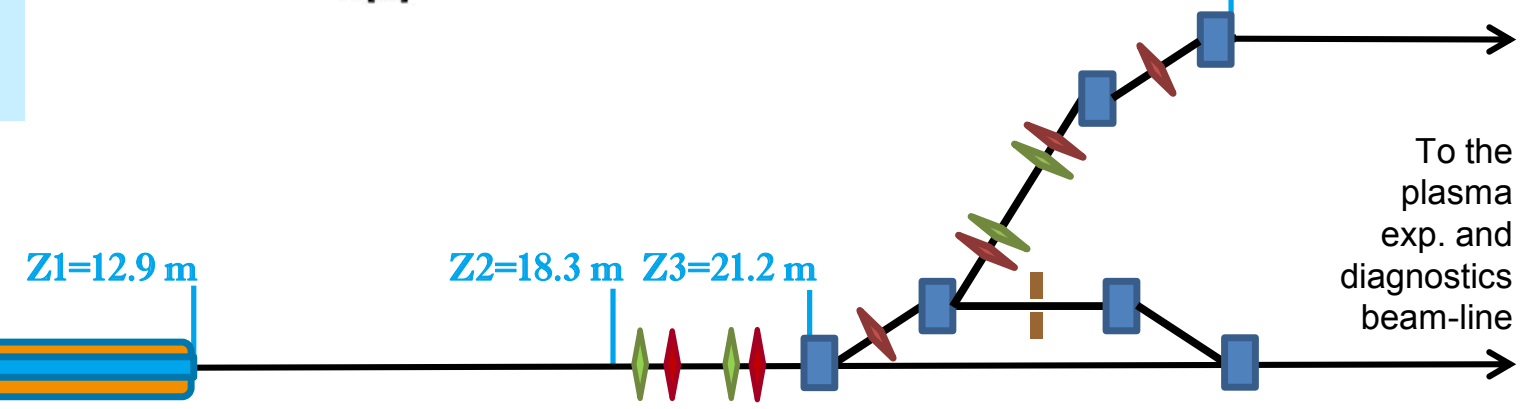




\section{VELOCITY BUNCHING + POSITIVE R56}
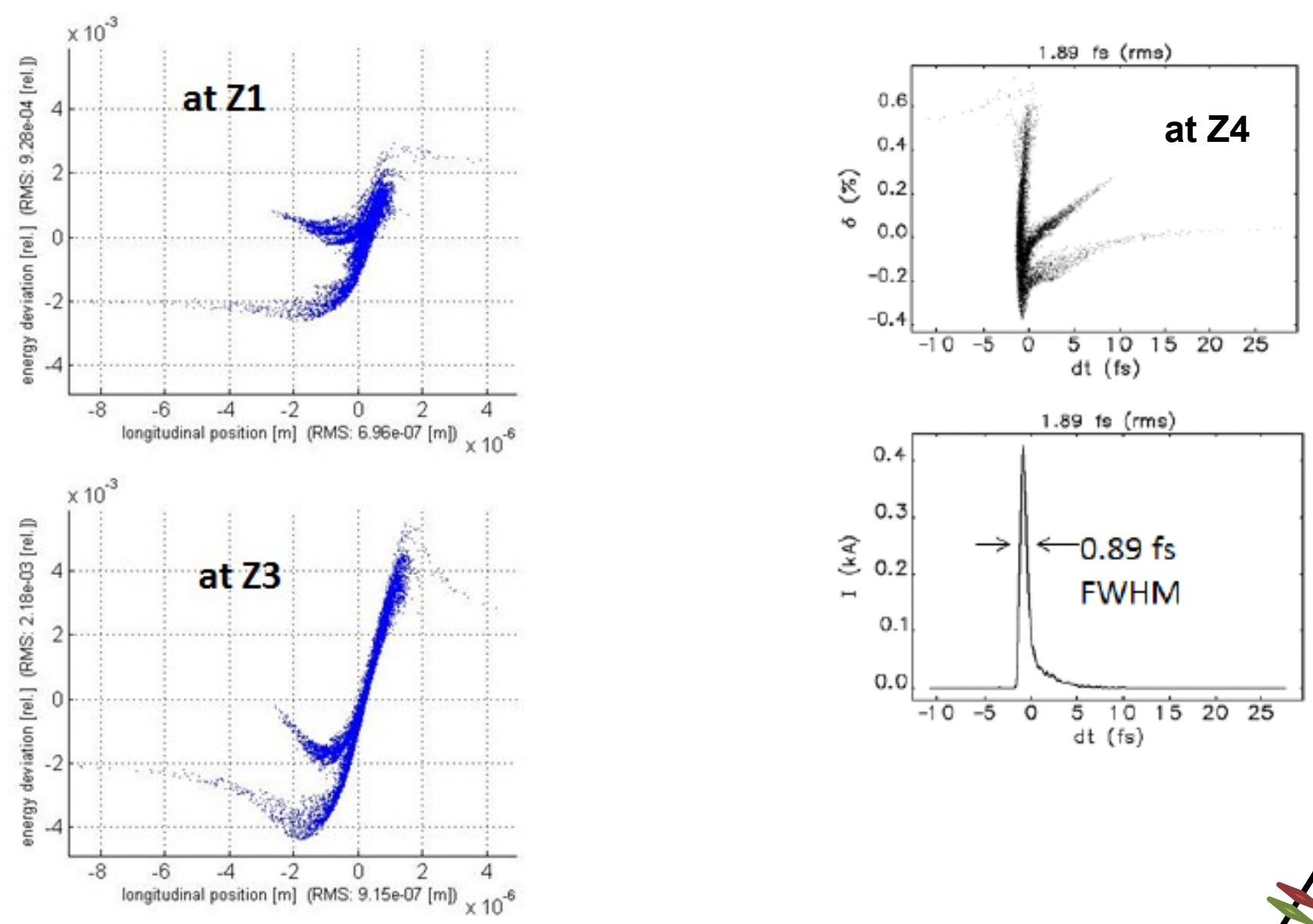

To the medical imaging beam-line

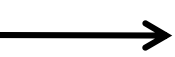

To the plasma exp. and

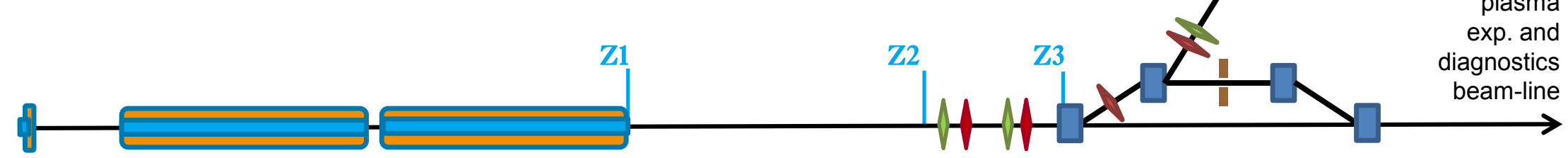




\section{MAGNETIC COMPRESSION WITH THE SLIT CUT}

- P. Emma et al, TUBIS01, Proc. FEL2004.

- P. Emma et al., PRL 927 (2004).

- S. Di Mitri et al., PRSTAB 16, 042801 (2013).
Pro: very high current+ short beam, non-linearity cut out, distributed RF phase tolerances

Contra: charge loss

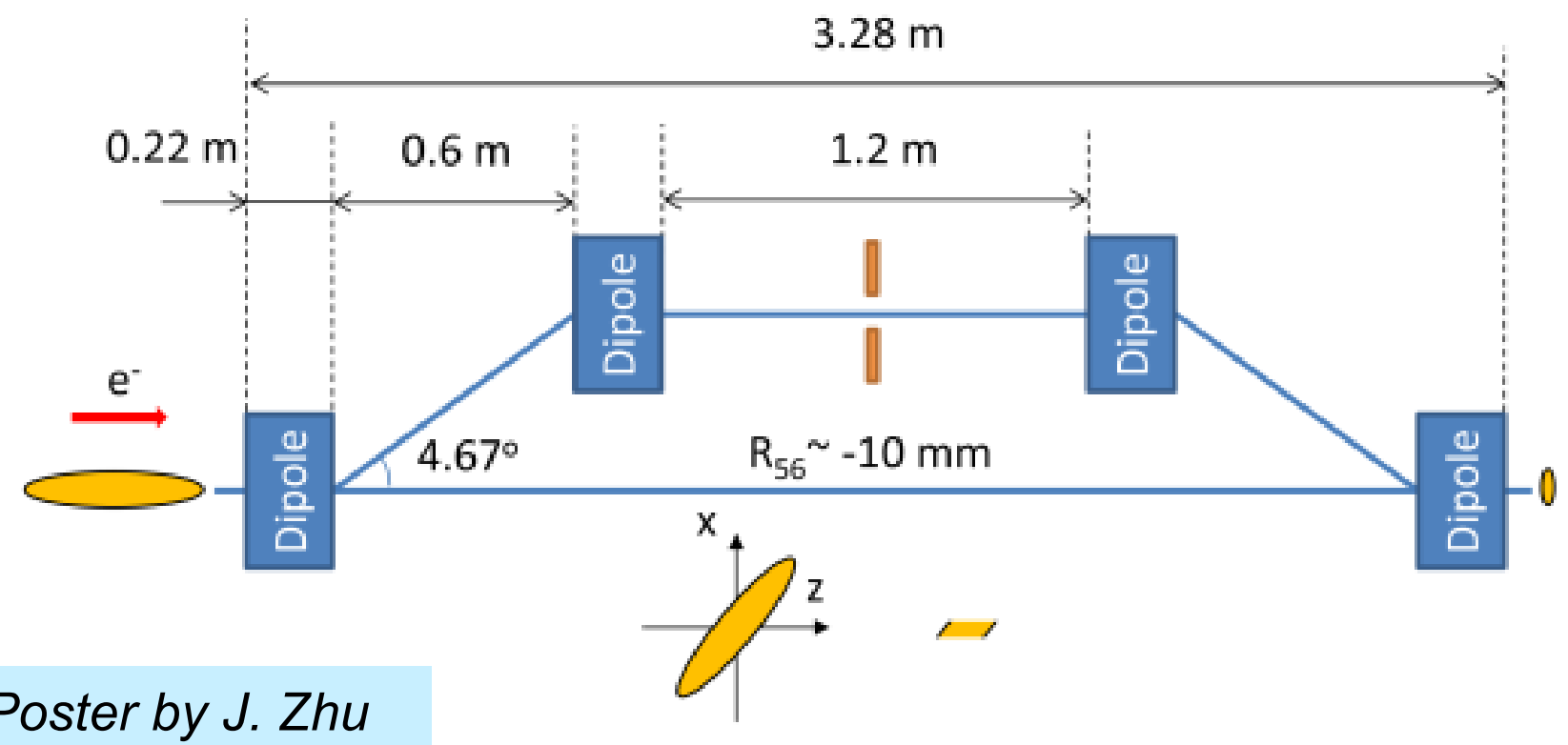

in WG4. 


\section{MAGNETIC COMPRESSION WITH THE SLIT}

J. Zhu et al,, MOPWA042, Proc. IPAC 2015.

J. Zhu et al., submitted paper
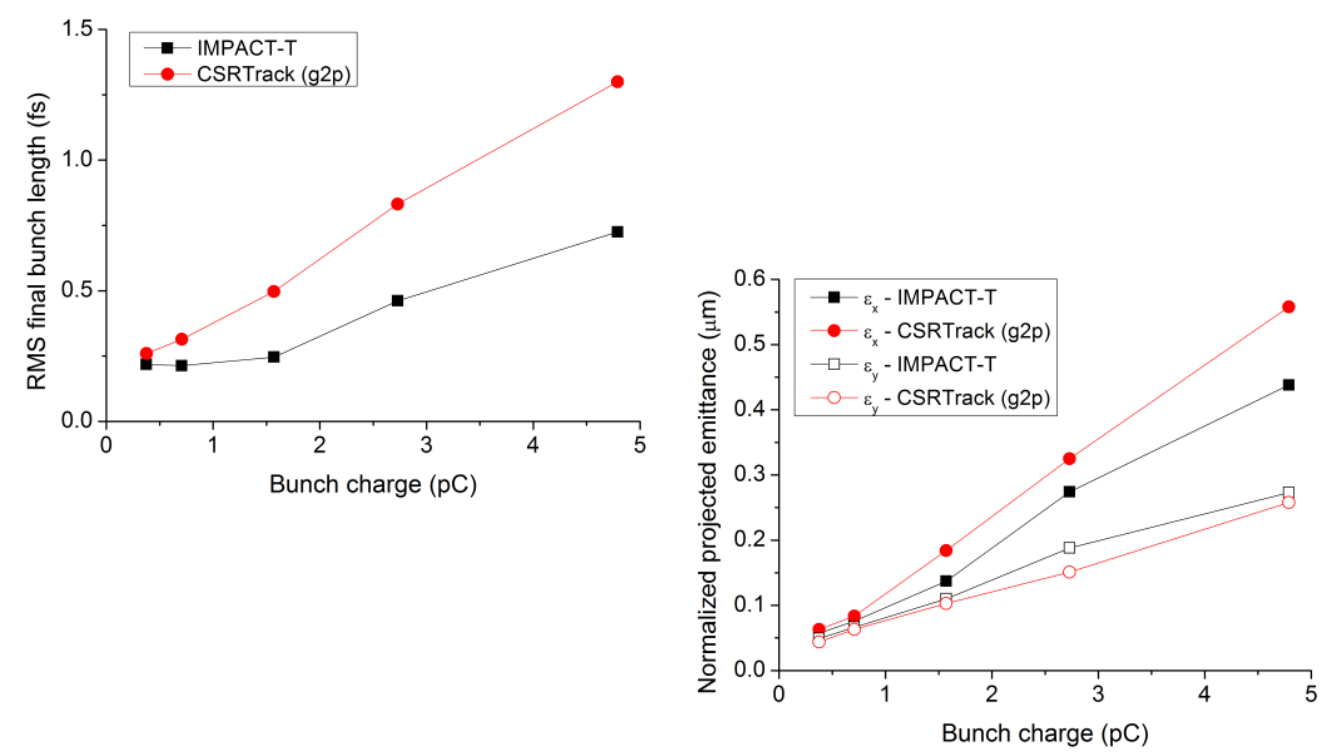
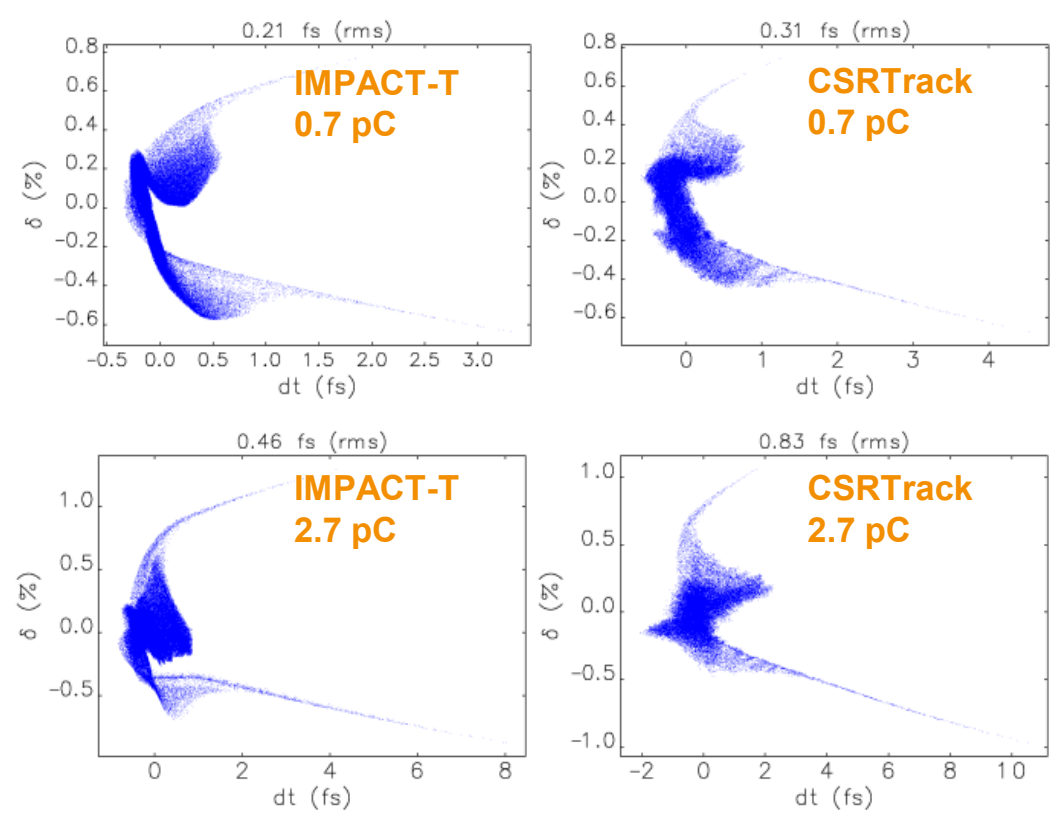

Bunch parameters before and after compression (slit width $0.4 \mathrm{~mm}$ )

\begin{tabular}{|c|c|c|c|c|c|}
\hline Bunch charge $(\mathrm{pC})$ & 10 & 20 & 50 & 100 & 200 \\
\hline RMS bunch duration $(\mathrm{ps})$ & 2.0 & 2.1 & 2.2 & 2.5 & 2.8 \\
\hline Normalized slice emittance $(\mu \mathrm{m})$ & 0.04 & 0.06 & 0.10 & 0.15 & 0.26 \\
\hline Final bunch charge $(\mathrm{pC})$ & 0.4 & 0.7 & 1.6 & 2.7 & 4.8 \\
\hline
\end{tabular}

Poster by J. Zhu in WG4. 


\section{MAGNETIC COMPRESSION WITH THE SLIT (HYBRID)}

Velocity bunching $\rightarrow$ Acceleration $\rightarrow$ Magnetic bunch compressor with slit cut
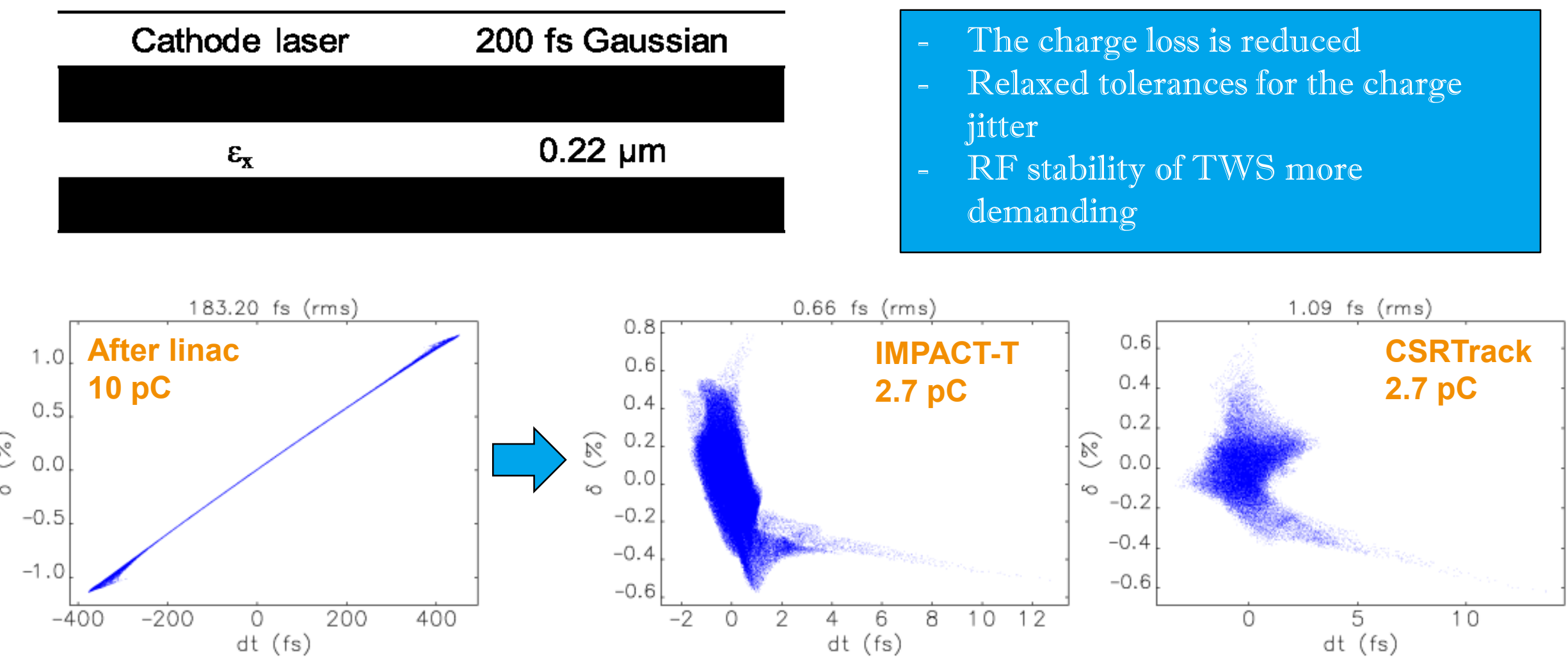

J. Zhu et al,, MOPWA042, Proc. IPAC 2015.

J. Zhu et al, submitted paper

Poster by J. Zhu in WG4. 


\section{SUMMARY TABLE WORKING POINTS}

\begin{tabular}{|c|c|c|c|c|c|}
\hline & WP3 (vb) & WP2 (vb) & WP (BC) & WP (VB+BC) & \multirow{10}{*}{$\begin{array}{l}{ }^{*} \text { Peak current } \\
I=\left(Q_{\text {TOT }}\right) /\left(3.5^{*} t_{\text {RMS }}\right) \\
{ }^{* *} \text { Local peak } \\
\text { current: } \\
\text { I=( }\left(Q_{\text {FWHM }}\right) /\left(t_{\text {FWHM }}\right) \\
{ }^{* * *} \text { Brightness } \\
B=I /\left(\gamma^{2} \varepsilon_{x} \varepsilon_{y}\right)\end{array}$} \\
\hline$Q$ final $[p C]$ & 0.5 & 0.5 & 0.7 & 2.8 & \\
\hline$Q$ initial $[p C]$ & 0.5 & 0.5 & 20 & 10 & \\
\hline tRMS [fs] & 2.486 & 2.321 & 0.21 & 0.66 & \\
\hline tFWHM [fs] & 4.1 & 2.777 & 0.22 & 1.54 & \\
\hline $\mathrm{E}[\mathrm{MeV}]$ & 110.9 & 110.9 & 100.2 & 101.6 & \\
\hline$\Delta \mathrm{E} / \mathrm{E}$ & $0.3 \%$ & $0.09 \%$ & $0.2 \%$ & $0.2 \%$ & \\
\hline xRMS [mm] & 0.009 & 0.152 & 0.059 & 0.087 & \\
\hline yRMS [mm] & 0.009 & 0.152 & 0.057 & 0.090 & \\
\hline $\mathrm{n} \varepsilon x[\mu \mathrm{m}]$ & 0.054 & 0.072 & 0.076 & 0.22 & \\
\hline nعy $[\mu \mathrm{m}]$ & 0.054 & 0.073 & 0.066 & 0.21 & \\
\hline $\begin{array}{l}\text { Peak current } \\
{[A]^{*}}\end{array}$ & 57 & 62 & 950 & 1200 & \\
\hline $\begin{array}{l}\text { Local peak } \\
{\text { current }[A]^{\star *}}^{*}\end{array}$ & 85 & 111 & 1730 & 1490 & \\
\hline $\mathrm{B}\left[\mathrm{A} / \mathrm{m}^{2}\right]^{* * *}$ & $1.97 * 10^{16}$ & $1.16 * 10^{16}$ & $1.89 * 10^{17}$ & $2.60 * 10^{16}$ & \\
\hline
\end{tabular}




\section{TOLERANCES}

\begin{tabular}{|c|c|c|c|c|c|c|c|c|c|}
\hline \multirow{2}{*}{ Jitter source } & \multirow{2}{*}{ Unit } & \multicolumn{4}{|c|}{ Sensitivity for 10 -fs BATJ } & \multicolumn{4}{|c|}{ RMS Tolerance } \\
\hline & & $\begin{array}{c}0.7 \mathrm{pC} \\
B C \\
\end{array}$ & $\begin{array}{c}2.7 \mathrm{pC} \\
B C \\
\end{array}$ & $\begin{array}{l}2.7 \mathrm{pC} \\
\text { Hybrid }\end{array}$ & $\begin{array}{c}0.5 \mathrm{pC} \\
V B \\
B\end{array}$ & $\begin{array}{c}0.7 \mathrm{pC} \\
B C \\
\end{array}$ & $\begin{array}{c}2.7 \mathrm{pC} \\
B C \\
\end{array}$ & $\begin{array}{l}2.7 \mathrm{pC} \\
\text { Hybrid }\end{array}$ & $\begin{array}{c}0.5 \mathrm{pC} \\
V B \\
\end{array}$ \\
\hline Laser-to-RF & fs & 42437.1 & 5949.7 & 159.8 & 125.0 & 200 & 200 & 50 & 50 \\
\hline Gun Charge & $\%$ & 5.8 & 1.6 & 301.6 & 1010.1 & 1.0 & $\begin{array}{c}0.3 \\
(1.0) \\
\end{array}$ & 4.0 & 4.0 \\
\hline Gun Phase & deg & 1.75 & 0.78 & 0.61 & 0.49 & 0.06 & 0.06 & 0.06 & 0.06 \\
\hline Gun Voltage & $\%$ & 0.61 & 1.14 & 0.72 & 0.40 & 0.06 & 0.06 & 0.06 & 0.06 \\
\hline TWS1 Phase & deg & 0.021 & 0.021 & 0.011 & 0.0098 & 0.013 & $\begin{array}{c}0.013 \\
(0.010) \\
\end{array}$ & 0.009 & 0.008 \\
\hline TWS1 Voltage & $\%$ & 0.055 & 0.055 & 0.073 & 0.10 & 0.013 & $\begin{array}{c}0.013 \\
(0.010)\end{array}$ & 0.009 & 0.008 \\
\hline TWS2 Phase & deg & 0.022 & 0.022 & 0.13 & 42.1 & 0.013 & $\begin{array}{c}0.013 \\
(0.010) \\
\end{array}$ & 0.011 & 0.060 \\
\hline TWS2 Voltage & $\%$ & 0.064 & 0.064 & 0.040 & 1.2 & 0.013 & $\begin{array}{c}0.013 \\
(0.010)\end{array}$ & 0.011 & 0.060 \\
\hline Magnetic field & $\%$ & 0.030 & 0.030 & 0.030 & 1 & 0.01 & 0.01 & 0.01 & 1 \\
\hline Total BATJ & fs & 1 & 1 & 1 & 1 & 9.91 & $\begin{array}{c}9.93 \\
(10.01)\end{array}$ & 9.94 & 9.34 \\
\hline
\end{tabular}

J. Zhu et al., WEPMA031, Proc. of IPAC 2015. 


\section{Summary and conclusions}

$>$ The layout and the goals of the SINBAD linac have been illustrated.

$>$ The procurement of the baseline layout is ongoing.

$>$ We have reviewed the bunch compression techniques that will be feasible.

$>$ Simulations confirmed us that the goal parameters concerning the beam quality are achievable.

$>$ The fulfillment of the tolerances for the arrival time jitter is challenging but reasonable.

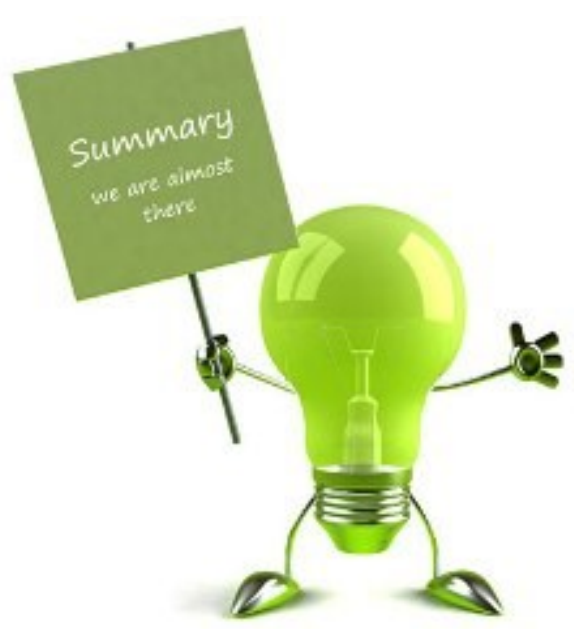




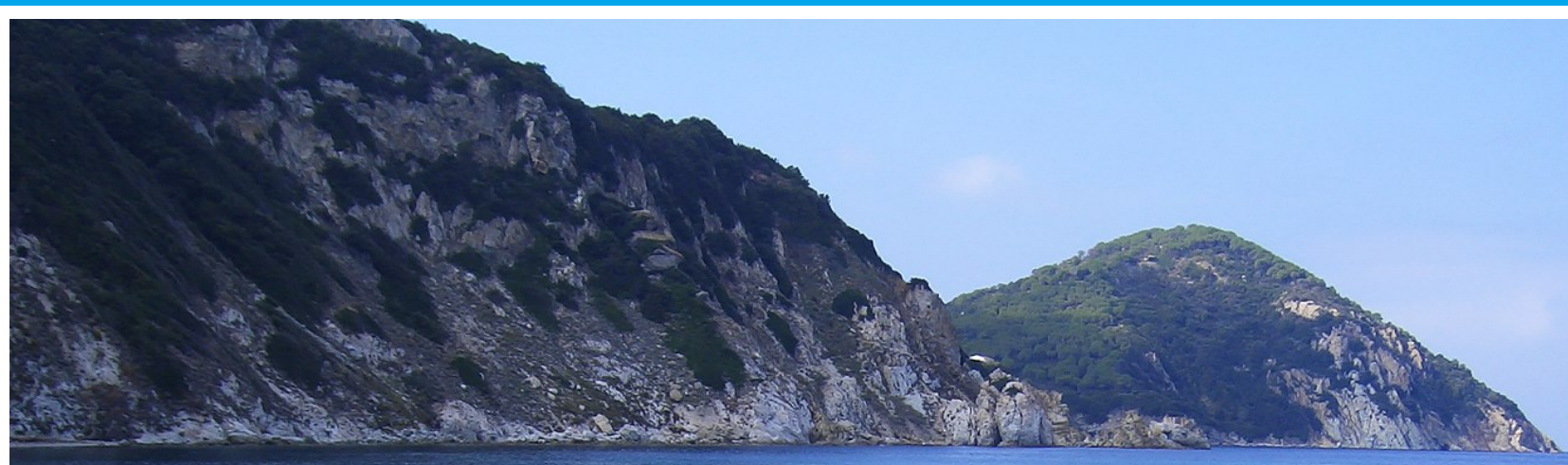

\section{Thank you for the attention!}

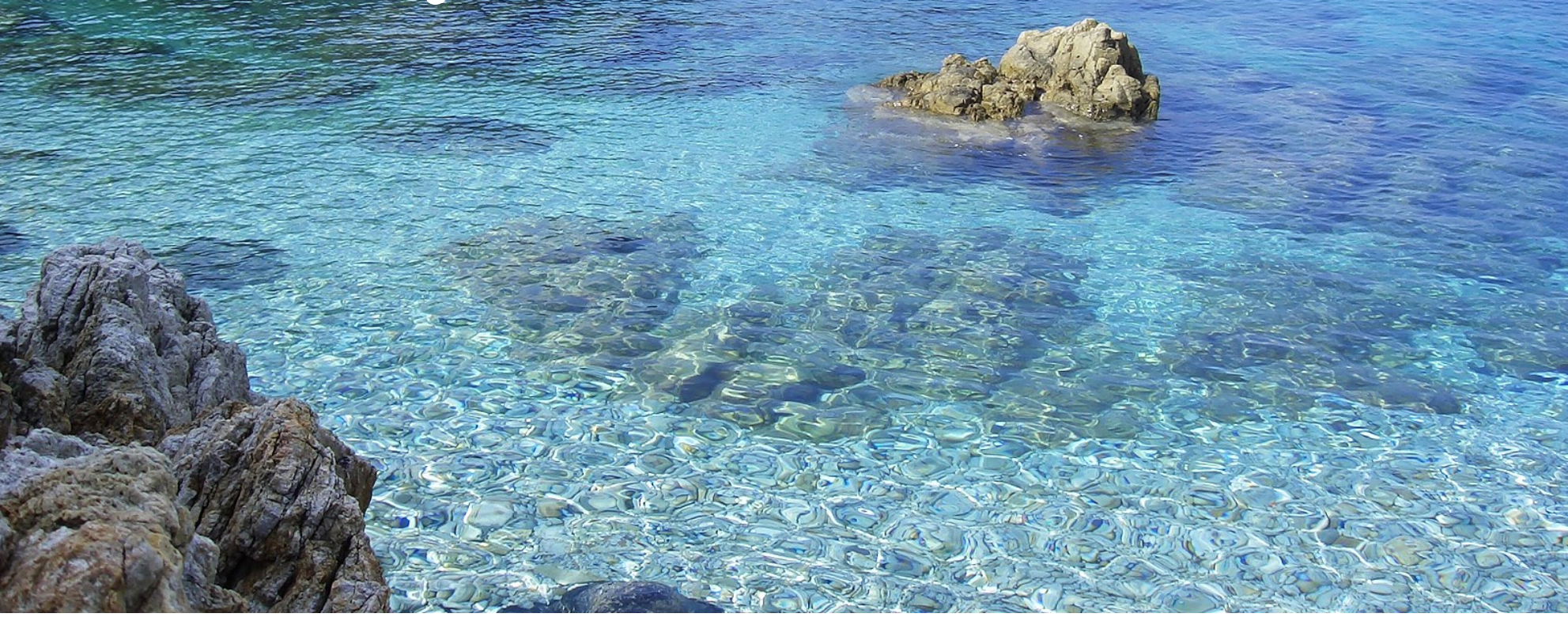




\section{Backup}

